\title{
GROWTH OF $\varphi$-ORDER SOLUTIONS OF LINEAR DIFFERENTIAL EQUATIONS WITH MEROMORPHIC COEFFICIENTS ON THE COMPLEX PLANE
}

\author{
Mohamed Abdelhak Kara, Benharrat Belaïdi ${ }^{1}$ \\ Department of Mathematics, Laboratory of Pure and Applied Mathematics, \\ University of Mostaganem (UMAB), B. P. 227 Mostaganem, Algeria \\ ${ }^{1}$ benharrat.belaidi@univ-mosta.dz
}

\begin{abstract}
In this paper, we study the growth of solutions of higher order linear differential equations with meromorphic coefficients of $\varphi$-order on the complex plane. By considering the concepts of $\varphi$-order and $\varphi$-type, we will extend and improve many previous results due to Chyzhykov-Semochko, Belaïdi, Cao-Xu-Chen, Kinnunen.
\end{abstract}

Keywords: Linear differential equations, Entire function, Meromorphic function, $\varphi$-order, $\varphi$-type.

\section{Introduction}

Let us consider the following linear differential equations

$$
\begin{gathered}
f^{(k)}+A_{k-1}(z) f^{(k-1)}+\cdots+A_{0}(z) f=0, \\
f^{(k)}+A_{k-1}(z) f^{(k-1)}+\cdots+A_{0}(z) f=F(z),
\end{gathered}
$$

where $k \geq 2, A_{0} \not \equiv 0$ and $F \not \equiv 0$. It is well-known that if the coefficients $A_{0}, A_{1}, \ldots, A_{k-1}$ and $F$ are entire functions, then all solutions of (1.1) and (1.2) are entire. The equation (1.1) has at least one solution of infinite order if some of coefficients are transcendental. For more details about the growth of solutions of equations (1.1) and (1.2), the reader can refer to [14]. In this paper, we use the standard notations of Nevanlinna value distribution theory of meromorphic functions (see $[10,14,18,22])$. The term meromorphic function throughout this paper means meromorphic in the whole complex plane $\mathbb{C}$. This will not be recalled in the next statements.

To study the growth of meromorphic functions, we recall the following definitions. For all $r \in \mathbb{R}$, we define $\exp _{1} r=\exp r=e^{r}$ and $\exp _{p+1} r=\exp \left(\exp _{p} r\right), p \in \mathbb{N}=\{1,2, \ldots\}$. Inductively, for all $r \in(0,+\infty)$ large enough, we define $\log _{1} r=\log r$ and $\log _{p+1} r=\log \left(\log _{p} r\right), p \in \mathbb{N}$. We also denote $\exp _{0} r=r=\log _{0} r, \exp _{-1} r=\log _{1} r$ and $\log _{-1} r=\exp _{1} r$.

Definition 1 [13]. The iterated p-order of a meromorphic function $f$ is defined by

$$
\rho_{p}(f):=\limsup _{r \rightarrow+\infty} \frac{\log _{p} T(r, f)}{\log r}, p \in \mathbb{N}
$$

where $T(r, f)$ is the Nevanlinna characteristic function of $f$. If $f$ is an entire function, then the iterated $p$-order is defined as

$$
\widetilde{\rho}_{p}(f):=\limsup _{r \rightarrow+\infty} \frac{\log _{p+1} M(r, f)}{\log r}=\rho_{p}(f),
$$

where $M(r, f)=\max \{|f(z)|:|z|=r\}$ is the maximum modulus of $f$. 
Note that $\rho_{1}(f)=\rho(f)$ is the usual order and $\rho_{2}(f)$ is the hyper-order.

Definition 2 [13]. The growth index of the iterated p-order of a meromorphic function $f$ is defined by

$i(f)= \begin{cases}0 & \text { if } f \text { is rational, } \\ \min \left\{j \in \mathbb{N}: \rho_{j}(f)<+\infty\right\} & \text { if } f \text { is transcendental and } \rho_{j}(f)<+\infty \text { for some } j \in \mathbb{N}, \\ +\infty & \text { if } \rho_{j}(f)=+\infty \text { for all } j \in \mathbb{N} .\end{cases}$

Historically, Bernal [4] was the first one who introduced the idea of the iterated order to study the growth of solutions of complex differential equations. In [13], Kinnunen considered the growth of solutions of equations (1.1) and (1.2) with entire coefficients of a finite iterated $p$-order and extended many previous results obtained for the usual order and the hyper-order.

Theorem A [13]. Let $A_{0}(z), \ldots, A_{k-1}(z)$ be entire functions such that $i\left(A_{0}\right)=p(0<p<\infty)$. If either $\max \left\{i\left(A_{j}\right): j=1,2, \ldots, k-1\right\}<p$ or $\max \left\{\rho_{p}\left(A_{j}\right): j=1,2, \ldots, k-1\right\}<\rho_{p}\left(A_{0}\right)$, then every solution $f \not \equiv 0$ of equation (1.1) satisfies $i(f)=p+1$ and $\rho_{p+1}(f)=\rho_{p}\left(A_{0}\right)$.

In [3], the second author has extended Theorem A when most of the coefficients $A_{0}(z), \ldots, A_{k-1}(z)$ have the same order by using the concept of iterated $p$-type as follows.

Theorem $\mathbf{B}[3]$. Let $A_{0}(z), \ldots, A_{k-1}(z)$ be entire functions, and let $i\left(A_{0}\right)=p(0<p<\infty)$. Assume that

$$
\max \left\{\rho_{p}\left(A_{j}\right): j=1,2, \ldots, k-1\right\} \leq \rho_{p}\left(A_{0}\right)=\rho \quad(0<\rho<+\infty)
$$

and

$$
\max \left\{\widetilde{\tau}_{p}\left(A_{j}\right): \rho_{p}\left(A_{j}\right)=\rho_{p}\left(A_{0}\right)\right\}<\widetilde{\tau}_{p}\left(A_{0}\right)=\tau \quad(0<\tau<+\infty)
$$

where

$$
\widetilde{\tau}_{p}(f)=\limsup _{r \rightarrow+\infty} \frac{\log _{p} M(r, f)}{r^{\rho_{p}(f)}} .
$$

Then, every solution $f \not \equiv 0$ of equation (1.1) satisfies $i(f)=p+1$ and $\rho_{p+1}(f)=\rho_{p}\left(A_{0}\right)=\rho$.

In [5], Cao- $\mathrm{Xu}-\mathrm{Chen}$ improved Theorems $\mathrm{A}$ and $\mathrm{B}$ by considering meromorphic coefficients instead of entire coefficients. In [16], Liu-Tu-Shi made a small modification in the original definition of $[p, q]$-order introduced by Juneja-Kapoor-Bajpai [11] in order to study the growth of entire solutions of equations (1.1) and (1.2). After that, $\mathrm{Li}$ and Cao [15] investigated the growth of meromorphic solutions of equations (1.1) and (1.2) with meromorphic coefficients of $[p, q]$-order which improved many results in $[3,5,13,16]$.

Definition $3[15,16]$. Let $p \geq q \geq 1$ be integers. The $[p, q]$-order of transcendental meromorphic function $f$ is defined by

$$
\rho_{[p, q]}(f)=\limsup _{r \rightarrow+\infty} \frac{\log _{p} T(r, f)}{\log _{q} r} .
$$

If $f$ is transcendental entire function, then

$$
\rho_{[p, q]}(f)=\limsup _{r \rightarrow+\infty} \frac{\log _{p+1} M(r, f)}{\log _{q} r} .
$$

Note that $\rho_{[p, 1]}(f)=\rho_{p}(f)$ is the iterated $p$-order $($ see $[13,14])$. 
Definition 4 [15]. The $[p, q]$-type of a meromorphic function $f$ with $[p, q]$-order $\rho_{[p, q]}(f) \in$ $(0,+\infty)$ is defined by

$$
\tau_{[p, q]}(f)=\limsup _{r \rightarrow+\infty} \frac{\log _{p-1} T(r, f)}{\left(\log _{q-1} r\right)^{\rho[p, q]}(f)} .
$$

Definition 5 [15]. Let $p \geq q \geq 1$ be integers. The $[p, q]$-convergence exponent of the sequence of zeros of a meromorphic function $f$ is defined by

$$
\lambda_{[p, q]}(f)=\limsup _{r \rightarrow+\infty} \frac{\log _{p} N(r, 1 / f)}{\log _{q} r},
$$

where $N(r, 1 / f)$ is the integrated counting function of zeros of $f$ in $\{z:|z| \leq r\}$. Similarly, the $[p, q]$-convergence exponent of the sequence of distinct zeros of $f$ is defined by

$$
\bar{\lambda}_{[p, q]}(f)=\limsup _{r \rightarrow+\infty} \frac{\log _{p} \bar{N}(r, 1 / f)}{\log _{q} r},
$$

where $\bar{N}(r, 1 / f)$ is the integrated counting function of distinct zeros of $f$ in $\{z:|z| \leq r\}$.

Here, we give two results due to Li-Cao in [15] concerning the growth of meromorphic solutions of equations (1.1) and (1.2) when the coefficients are meromorphic functions of $[p, q]$-order.

Theorem $\mathbf{C}[15]$. Let $A_{0}, A_{1}, \ldots, A_{k-1}$ be meromorphic functions such that

$$
\max \left\{\rho_{[p, q]}\left(A_{j}\right), \lambda_{[p, q]}\left(\frac{1}{A_{0}}\right): j=1, \ldots, k-1\right\}<\rho_{[p, q]}\left(A_{0}\right)<+\infty .
$$

Then every meromorphic solution $f \not \equiv 0$ whose poles are of uniformly bounded multiplicities of equation (1.1) satisfies $\rho_{[p+1, q]}(f)=\rho_{[p, q]}\left(A_{0}\right)$.

If there exist some other coefficients $A_{j}(j=1, \ldots, k-1)$ having the same $[p, q]$-order as $A_{0}$, then we have the following result.

Theorem D [15]. Let $A_{0}, A_{1}, \ldots, A_{k-1}$ be meromorphic functions such that $\lambda_{[p, q]}\left(1 / A_{0}\right)<$ $\rho_{[p, q]}\left(A_{0}\right)$ and

$$
\begin{gathered}
\max \left\{\rho_{[p, q]}\left(A_{j}\right): j=1, \ldots, k-1\right\}=\rho_{[p, q]}\left(A_{0}\right)<+\infty, \\
\max \left\{\tau_{[p, q]}\left(A_{j}\right): \rho_{[p, q]}\left(A_{j}\right)=\rho_{[p, q]}\left(A_{0}\right)>0, j=1, \ldots, k-1\right\}<\tau_{[p, q]}\left(A_{0}\right) .
\end{gathered}
$$

Then any non-zero meromorphic solution $f$ whose poles are of uniformly bounded multiplicities of (1.1) satisfies $\rho_{[p+1, q]}(f)=\rho_{[p, q]}\left(A_{0}\right)$.

It is clear that Theorem $\mathrm{C}$ and Theorem D improve respectively Theorem A and Theorem B from entire coefficients of iterated $p$-order to meromorphic coefficients of $[p, q]$-order. Recently, Chyzhykov and Semochko [7] showed that both definitions of iterated $p$-order and $[p, q]$-order have the disadvantage that they do not cover arbitrary growth (see [7, Example 1.4]). They introduced more general scale to measure the growth of entire solutions of equation (1.1) called the $\varphi$-order (see [20]).

Definition 6 [7]. Let $\varphi$ be an increasing unbounded function on $[1,+\infty)$. The $\varphi$-orders of a meromorphic function $f$ are defined by

$$
\rho_{\varphi}^{0}(f)=\limsup _{r \rightarrow+\infty} \frac{\varphi\left(e^{T(r, f)}\right)}{\log r}, \quad \rho_{\varphi}^{1}(f)=\limsup _{r \rightarrow+\infty} \frac{\varphi(T(r, f))}{\log r} .
$$

If $f$ is an entire function, then the $\varphi$-orders are defined by

$$
\tilde{\rho}_{\varphi}^{0}(f)=\limsup _{r \rightarrow+\infty} \frac{\varphi(M(r, f))}{\log r}, \quad \tilde{\rho}_{\varphi}^{1}(f)=\limsup _{r \rightarrow+\infty} \frac{\varphi(\log M(r, f))}{\log r} .
$$


Definition 7 [1]. Let $\varphi$ be an increasing unbounded function on $[1,+\infty)$. We define the $\varphi$-types of a meromorphic function $f$ with $\varphi$-order $\in(0,+\infty)$ by

$$
\tau_{\varphi}^{0}(f)=\limsup _{r \rightarrow+\infty} \frac{e^{\varphi\left(e^{T(r, f)}\right)}}{r^{\rho_{\varphi}^{0}(f)}}, \quad \tau_{\varphi}^{1}(f)=\limsup _{r \rightarrow+\infty} \frac{e^{\varphi(T(r, f))}}{r^{\rho_{\varphi}^{1}(f)}} .
$$

If $f$ is an entire function, then the $\varphi$-types are defined as

$$
\tilde{\tau}_{\varphi}^{0}(f)=\limsup _{r \rightarrow+\infty} \frac{e^{\varphi(M(r, f))}}{r^{\tilde{\rho}_{\varphi}^{0}(f)}}, \quad \tilde{\tau}_{\varphi}^{1}(f)=\limsup _{r \rightarrow+\infty} \frac{e^{\varphi(\log M(r, f))}}{r^{\tilde{\rho}_{\varphi}^{1}(f)}} .
$$

By symbol $\Phi$ we define the class of positive unbounded increasing functions on $[1,+\infty)$, such that $\varphi\left(e^{t}\right)$ grows slowly, i. e., $\forall c>0: \lim _{r \rightarrow+\infty} \frac{\varphi\left(e^{c t}\right)}{\varphi\left(e^{t}\right)}=1$.

Example 1. Let $f$ be a meromorphic function. One can see that $\varphi(r)=\log _{p} r,(p \geq 2)$ belongs to the class $\Phi$ and $\varphi(r)=\log r \notin \Phi$. Moreover, the $\rho_{\varphi}^{1}(f)$ order of the function $f$ coincides with its iterated $p$-order, i. e., $\rho_{\varphi}^{1}(f)=\rho_{p}(f)$. As a particular case, for $\varphi=\log _{2} \in \Phi$ we have $\rho_{\log _{2}}^{0}(f)=\rho_{1}(f)$ and $\rho_{\log _{2}}^{1}(f)=\rho_{2}(f)$ which are respectively the usual order and the hyper-order of $f$.

The following result due to Chyzhykov-Semochko [7] investigates the growth of entire solutions of equation (1.1) when the coefficients are entire functions of $\varphi$-order.

Theorem $\mathbf{E}$ [7]. Let $\varphi \in \Phi$ and $A_{0}, A_{1}, \ldots, A_{k-1}$ be entire functions such that

$$
\max \left\{\rho_{\varphi}^{0}\left(A_{j}\right), j=1, \ldots, k-1\right\}<\rho_{\varphi}^{0}\left(A_{0}\right) .
$$

Then every solution $f \not \equiv 0$ of $(1.1)$ satisfies $\rho_{\varphi}^{1}(f)=\rho_{\varphi}^{0}\left(A_{0}\right)$.

We recall that the linear measure of a set $E \subset(0,+\infty)$ is defined by

$$
m(E)=\int_{0}^{+\infty} \chi_{E}(t) d t
$$

and the logarithmic measure of a set $F \subset(1,+\infty)$ is defined by

$$
\operatorname{lm}(F)=\int_{1}^{+\infty} \frac{\chi_{F}(t)}{t} d t
$$

where $\chi_{A}$ is the characteristic function of a set $A$. The upper density of a set $E \subset(0,+\infty)$ is defined by

$$
\overline{\operatorname{dens}} E=\limsup _{r \rightarrow+\infty} \frac{m(E \cap[0, r])}{r} .
$$

The upper logarithmic density of a set $F \subset(1,+\infty)$ is defined by

$$
\overline{\log d e n s} F=\limsup _{r \rightarrow+\infty} \frac{\operatorname{lm}(F \cap[1, r])}{\log r} .
$$

Definition 8 [10, 22]. For $a \in \overline{\mathbb{C}}=\mathbb{C} \cup\{\infty\}$, the deficiency of a with respect to a meromorphic function $f$ is defined as

$$
\begin{gathered}
\delta(a, f)=\liminf _{r \rightarrow+\infty} \frac{m(r, 1 /(f-a))}{T(r, f)}=1-\limsup _{r \rightarrow+\infty} \frac{N(r, 1 /(f-a))}{T(r, f)}, \quad a \neq \infty, \\
\delta(\infty, f)=\liminf _{r \rightarrow+\infty} \frac{m(r, f)}{T(r, f)}=1-\limsup _{r \rightarrow+\infty} \frac{N(r, f)}{T(r, f)} .
\end{gathered}
$$


Recently, the second author has studied the growth of entire solutions of equation (1.1) when the coefficients are entire functions of $\varphi$-order and obtained the following results.

Theorem $\mathbf{F}$ [2]. Let $G$ be a set of complex numbers $z$ satisfying $\overline{\log d e n s}\{|z|: z \in G\}>0$. Let $\varphi \in \Phi$ and let $A_{0}, A_{1}, \ldots, A_{k-1}$ be entire functions satisfying

$$
\max \left\{\rho_{\varphi}^{0}\left(A_{j}\right): j=0,1, \ldots, k-1\right\} \leq \alpha \quad(0<\alpha<+\infty) .
$$

Suppose, there exists a real number $\beta$ satisfies $0<\beta<\alpha$ such that for any given $\varepsilon(0<2 \varepsilon<\alpha-\beta)$, we have

$$
T\left(r, A_{0}\right) \geq \log \left(\varphi^{-1}((\alpha-\varepsilon) \log r)\right)
$$

and

$$
T\left(r, A_{j}\right) \leq \log \left(\varphi^{-1}(\beta \log r)\right), \quad j=1, \ldots, k-1
$$

as $|z| \rightarrow+\infty$ for $z \in G$. Then every non-zero solution $f$ of equation (1.1) satisfies $\rho_{\varphi}^{1}(f)=\alpha$.

Theorem G [1]. Let $A_{0}(z), \ldots, A_{k-1}(z)$ be entire functions, and let $\varphi \in \Phi$. Assume that

$$
\max \left\{\tilde{\rho}_{\varphi}^{0}\left(A_{j}\right): j=1, \ldots, k-1\right\} \leq \tilde{\rho}_{\varphi}^{0}\left(A_{0}\right)=\rho<+\infty \quad(0<\rho<+\infty)
$$

and

$$
\max \left\{\tilde{\tau}_{\varphi}^{0}\left(A_{j}\right): \tilde{\rho}_{\varphi}^{0}\left(A_{j}\right)=\tilde{\rho}_{\varphi}^{0}\left(A_{0}\right)\right\}<\tilde{\tau}_{\varphi}^{0}\left(A_{0}\right)=\tau \quad(0<\tau<+\infty) .
$$

Then every solution $f \not \equiv 0$ of (1.1) satisfies $\tilde{\rho}_{\varphi}^{1}(f)=\tilde{\rho}_{\varphi}^{0}\left(A_{0}\right)$.

\section{Main results}

The aim of this paper is to investigate the growth of meromorphic solutions of equations (1.1) and (1.2) with meromorphic coefficients of finite $\varphi$-order. By using the concept of $\varphi$-order, we can cover arbitrary growth of solutions of equations (1.1) and (1.2) which improves several results in $[1-3,5,7,13]$. To do that, we firstly introduce the following quantities by an analogous manner with the definitions of the $\varphi$-orders.

Definition 9. Let $\varphi$ be an increasing unbounded function on $[1,+\infty)$. We define the $\varphi$ convergence exponents of the sequence of zeros of a meromorphic function $f$ by

$$
\lambda_{\varphi}^{0}(f)=\limsup _{r \rightarrow+\infty} \frac{\varphi\left(e^{N(r, 1 / f)}\right)}{\log r}, \quad \lambda_{\varphi}^{1}(f)=\limsup _{r \rightarrow+\infty} \frac{\varphi(N(r, 1 / f))}{\log r} .
$$

Similarly, the notations $\bar{\lambda}_{\varphi}^{0}(f)$ and $\bar{\lambda}_{\varphi}^{1}(f)$ can be used to denote the $\varphi$-convergence exponents of the sequence of distinct zeros of $f$.

Now, we list our main results.

Theorem 1. Let $\varphi \in \Phi$ and $A_{0}, A_{1}, \ldots, A_{k-1}$ be meromorphic functions. Suppose, there exists one coefficient $A_{s}(s \in\{0,1, \ldots, k-1\})$ such that

$$
\max \left\{\rho_{\varphi}^{0}\left(A_{j}\right), \lambda_{\varphi}^{0}\left(\frac{1}{A_{s}}\right): j=0,1, \ldots, k-1(j \neq s)\right\}<\rho_{\varphi}^{0}\left(A_{s}\right)<+\infty .
$$

Then every transcendental meromorphic solution $f$ whose poles are of uniformly bounded multiplicities of (1.1) satisfies

$$
\rho_{\varphi}^{1}(f) \leq \rho_{\varphi}^{0}\left(A_{s}\right) \leq \rho_{\varphi}^{0}(f) .
$$

Furthermore, if all solutions of (1.1) are meromorphic solutions, then there is at least one meromorphic solution, say $f_{1}$, verifies $\rho_{\varphi}^{1}\left(f_{1}\right)=\rho_{\varphi}^{0}\left(A_{0}\right)$. 
Remark 1. By setting $\varphi(r)=\log _{p+1} r(p \geq 1)$ in Theorem 1, we obtain Theorem 2.2 in [5].

Theorem 2. Let $\varphi \in \Phi$ and $A_{0}, A_{1}, \ldots, A_{k-1}$ be meromorphic functions such that

$$
\max \left\{\lambda_{\varphi}^{0}\left(\frac{1}{A_{0}}\right), \rho_{\varphi}^{0}\left(A_{j}\right): j=1, \ldots, k-1\right\}<\rho_{\varphi}^{0}\left(A_{0}\right)<+\infty .
$$

Then every non-zero meromorphic solution $f$ whose poles are of uniformly bounded multiplicities of (1.1) satisfies $\rho_{\varphi}^{1}(f)=\rho_{\varphi}^{0}\left(A_{0}\right)$.

Remark 2. Clearly, Theorem 2 is an extension of Theorem $\mathrm{E}$ from entire solutions of equation (1.1) to the case of meromorphic solutions of equation (1.1) with meromorphic coefficients instead of entire coefficients. Furthermore, by setting $\varphi(r)=\log _{p+1} r(p \geq 1)$ in Theorem 2, we obtain Theorem A when the coefficients of (1.1) are entire functions.

If there exist some other coefficients $A_{j}(j=1, \ldots, k-1)$ having the same $\varphi$-order as $A_{0}$, then we have the following result.

Theorem 3. Let $\varphi \in \Phi$ and $A_{0}, A_{1}, \ldots, A_{k-1}$ be meromorphic functions such that $\lambda_{\varphi}^{0}\left(1 / A_{0}\right)<\rho_{\varphi}^{0}\left(A_{0}\right)$ and

$$
\begin{gathered}
\max \left\{\rho_{\varphi}^{0}\left(A_{j}\right): j=1, \ldots, k-1\right\} \leq \rho_{\varphi}^{0}\left(A_{0}\right)=\rho_{0}<+\infty \\
\max \left\{\tau_{\varphi}^{0}\left(A_{j}\right): \rho_{\varphi}^{0}\left(A_{j}\right)=\rho_{\varphi}^{0}\left(A_{0}\right)>0, j=1, \ldots, k-1\right\}<\tau_{\varphi}^{0}\left(A_{0}\right)=\tau_{0}\left(0<\tau_{0}<+\infty\right) .
\end{gathered}
$$

Then any non-zero meromorphic solution $f$ whose poles are of uniformly bounded multiplicities of (1.1) satisfies $\rho_{\varphi}^{1}(f)=\rho_{\varphi}^{0}\left(A_{0}\right)$.

Remark 3. Namely, Theorem 3 extends Theorem G from entire solutions of equation (1.1) to meromorphic solutions. Furthermore, by setting $\varphi(r)=\log _{p+1} r(p \geq 1)$ in Theorem 3, we obtain Theorem 2.1 in [5] and Theorem B when the coefficients of (1.1) are entire functions.

Theorem 4. Let $\varphi \in \Phi$ and $A_{0}, A_{1}, \ldots, A_{k-1}, F \not \equiv 0$ be meromorphic functions such that $\lambda_{\varphi}^{0}\left(1 / A_{0}\right)<\rho_{\varphi}^{0}\left(A_{0}\right)$ and

$$
\max \left\{\rho_{\varphi}^{1}(F), \rho_{\varphi}^{0}\left(A_{j}\right): j=1, \ldots, k-1\right\}<\rho_{\varphi}^{0}\left(A_{0}\right)<+\infty .
$$

Then every meromorphic solution $f$ whose poles are of uniformly bounded multiplicities of (1.2) satisfies

$$
\bar{\lambda}_{\varphi}^{1}(f)=\lambda_{\varphi}^{1}(f)=\rho_{\varphi}^{1}(f)=\rho_{\varphi}^{0}\left(A_{0}\right)
$$

with at most one exceptional solution $f_{0}$ satisfying $\rho_{\varphi}^{1}\left(f_{0}\right)<\rho_{\varphi}^{0}\left(A_{0}\right)$.

Remark 4. Theorem 4 is a counterpart of Theorem 1.6 in [15]. Moreover, if we choose $\varphi(r)=$ $\log _{p+1} r(p \geq 1)$ in Theorem 4, then we obtain a special case of Theorem 2.6 in [21].

Theorem 5. Let $\varphi \in \Phi$ and $A_{0}, A_{1}, \ldots, A_{k-1}, F \not \equiv 0$ be meromorphic functions such that

$$
\max \left\{\rho_{\varphi}^{0}\left(A_{j}\right): j=0, \ldots, k-1\right\}<\rho_{\varphi}^{1}(F) .
$$

If all solutions $f$ of (1.2) are meromorphic functions whose poles are of uniformly bounded multiplicities, then there holds $\rho_{\varphi}^{1}(f)=\rho_{\varphi}^{1}(F)$ for all solutions of (1.2).

Remark 5. Theorem 5 is a counterpart of Theorem 1.7 in [15]. Furthermore, if we choose $\varphi(r)=\log _{p+1} r(p \geq 1)$ in Theorem 5, then we obtain a special case in [13, Remark 4.1, p. 399] when the coefficients of equation (1.1) are entire functions. 
Theorem 6. Let $G \subset(1,+\infty)$ be a set of complex numbers $z$ satisfying

$$
\overline{\log \operatorname{dens}}\{|z|: z \in G\}>0 .
$$

Let $\varphi \in \Phi$ and $A_{0}, A_{1}, \ldots, A_{k-1}$ be meromorphic functions satisfying $\delta\left(\infty, A_{0}\right)=\delta>0$ and

$$
\max \left\{\rho_{\varphi}^{0}\left(A_{j}\right): j=0,1, \ldots, k-1\right\} \leq \alpha \quad(0<\alpha<+\infty) .
$$

Suppose, there exists a real number $\beta$ satisfies $0<\beta<\alpha$ such that for any given $\varepsilon(0<2 \varepsilon<\alpha-\beta)$, we have

$$
T\left(r, A_{0}\right) \geq \log \left(\varphi^{-1}((\alpha-\varepsilon) \log r)\right)
$$

and

$$
T\left(r, A_{j}\right) \leq \log \left(\varphi^{-1}(\beta \log r)\right), \quad j=1, \ldots, k-1
$$

as $|z|=r \rightarrow+\infty$ for $z \in G$. Then every non-zero meromorphic solution of equation (1.1) satisfies $\rho_{\varphi}^{1}(f)=\alpha$.

Remark 6. Theorem 6 extends Theorem $\mathrm{F}$ from entire solutions of equation (1.1) to meromorphic solutions.

Theorem 7. Let $G \subset(1,+\infty)$ be a set of complex numbers $z$ satisfying

$$
\overline{\log \operatorname{dens}}\{|z|: z \in G\}>0 .
$$

Let $\varphi \in \Phi$ and $A_{0}, A_{1}, \ldots, A_{k-1}, F \not \equiv 0$ be meromorphic functions satisfying

$$
\max \left\{\rho_{\varphi}^{0}\left(A_{j}\right): j=0,1, \ldots, k-1\right\}<\alpha \quad(0<\alpha<+\infty) .
$$

Suppose, there exists a real number $\beta$ satisfies $0<\beta<\alpha$ such that for any given $\varepsilon(0<2 \varepsilon<\alpha-\beta)$, we have

$$
\left|A_{0}(z)\right| \geq \varphi^{-1}((\alpha-\varepsilon) \log r)
$$

and

$$
\left|A_{j}(z)\right| \leq \varphi^{-1}(\beta \log r), \quad j=1, \ldots, k-1
$$

as $|z|=r \rightarrow+\infty$ for $z \in G$. Then, the following conclusions hold

(i) If $\rho_{\varphi}^{1}(F) \geq \alpha$, then all meromorphic solutions $f$ whose poles are of uniformly bounded multiplicities of equation (1.2) satisfy $\rho_{\varphi}^{1}(f)=\rho_{\varphi}^{1}(F)$.

(ii) If $\rho_{\varphi}^{1}(F)<\alpha$, then every meromorphic solution $f$ whose poles are of uniformly bounded multiplicities of (1.2) satisfies

$$
\bar{\lambda}_{\varphi}^{1}(f)=\lambda_{\varphi}^{1}(f)=\rho_{\varphi}^{1}(f)=\alpha
$$

with at most one exceptional solution $f_{0}$ satisfying $\rho_{\varphi}^{1}\left(f_{0}\right)<\alpha$.

Remark 7. Clearly, Theorem 7 is an improvement of Theorem 1.15 in [2] from entire solutions of equation (1.2) to meromorphic solutions. Furthermore, Theorem 7 is a counterpart of Theorem 1.8 in $[15]$. 


\section{Preliminary lemmas}

Proposition 1 [7]. If $\varphi \in \Phi$, then

$$
\begin{gathered}
\forall m>0, \forall k \geq 0: \frac{\varphi^{-1}\left(\log x^{m}\right)}{x^{k}} \longrightarrow+\infty, \quad x \rightarrow+\infty, \\
\forall \delta>0: \frac{\log \varphi^{-1}((1+\delta) x)}{\log \varphi^{-1}(x)} \longrightarrow+\infty, \quad x \rightarrow+\infty .
\end{gathered}
$$

Remark 8 [7]. We can see that (3.2) implies that

$$
\forall c>0, \varphi(c t) \leq \varphi\left(t^{c}\right) \leq(1+o(1)) \varphi(t), \quad t \rightarrow+\infty
$$

Proposition 2 [7]. Let $\varphi \in \Phi$ and $f$ be an entire function. Then

$$
\rho_{\varphi}^{j}(f)=\tilde{\rho}_{\varphi}^{j}(f), \quad j=0,1 .
$$

Lemma 1 [6]. Let $f$ be a meromorphic solution of equation (1.1), suppose that not all coeffcients $A_{j}$ are constants. Given a real number $\gamma>1$, and denoting $T(r)=\sum_{j=0}^{k-1} T\left(r, A_{j}\right)$, then the inequalities

$$
\begin{aligned}
& \log m(r, f)<T(r)\{(\log r) \log T(r)\}^{\gamma} \quad \text { if } \quad s=0, \\
& \log m(r, f)<r^{2 s+\gamma-1} T(r)\{\log T(r)\}^{\gamma} \quad \text { if } \quad s>0
\end{aligned}
$$

take place outside of an exceptional set $E_{s}$ with $\int_{E_{s}} t^{s-1} d t<+\infty$.

Lemma 2 [8]. Let $f_{1}, f_{2}, \ldots, f_{k}$ be linearly independent meromorphic solutions of equation (1.1) with meromorphic coefficients $A_{0}, A_{1}, \ldots, A_{k-1}$. Then

$$
m\left(r, A_{j}\right)=O\left(\log \left(\max _{1 \leq i \leq k} T\left(r, f_{i}\right)\right)\right), \quad j=0,1, \ldots, k-1 .
$$

Lemma 3 [9]. Let $f$ be a transcendental meromorphic function and let $\alpha>1$ be a given constant. Then, there exists a set $E_{1} \subset(1,+\infty)$ with finite logarithmic measure and a constant $B_{\alpha}>0$ that depends only on $\alpha$ and $i, j(j>i \geq 0)$ such that for all $z$ satisfying $|z|=r \notin[0,1] \cup E_{1}$, we have

$$
\left|\frac{f^{(j)}(z)}{f^{(i)}(z)}\right| \leq B_{\alpha}\left\{\frac{T(\alpha r, f)}{r}\left(\log ^{\alpha} r\right) \log T(\alpha r, f)\right\}^{j-i} .
$$

Lemma 4 [12]. Let $f$ be a meromorphic function and $\varphi \in \Phi$. Then

$$
\rho_{\varphi}^{j}\left(f^{\prime}\right)=\rho_{\varphi}^{j}(f) \quad \text { for } \quad j=0,1
$$

Lemma $5[7,12]$. Let $\varphi \in \Phi$ and $f_{1}, f_{2}$ be two meromorphic functions. Then

(i) $\rho_{\varphi}^{j}\left(f_{1}+f_{2}\right) \leq \max \left\{\rho_{\varphi}^{j}\left(f_{1}\right), \rho_{\varphi}^{j}\left(f_{2}\right)\right\}$ and $\rho_{\varphi}^{j}\left(f_{1} f_{2}\right) \leq \max \left\{\rho_{\varphi}^{j}\left(f_{1}\right), \rho_{\varphi}^{j}\left(f_{2}\right)\right\}$ for $j=0,1$.

(ii) If $\rho_{\varphi}^{j}\left(f_{1}\right)<\rho_{\varphi}^{j}\left(f_{2}\right)$, then $\rho_{\varphi}^{j}\left(f_{1}+f_{2}\right)=\rho_{\varphi}^{j}\left(f_{1} f_{2}\right)=\rho_{\varphi}^{j}\left(f_{2}\right)$ for $j=0,1$. 
Lemma 6. Let $\varphi \in \Phi$ and $f$ be a meromorphic function. Then, for any set $E_{2} \subset[0,+\infty)$ with finite linear measure, there exists a sequence $\left\{r_{n}, r_{n} \notin E_{2}\right\}$ such that

$$
\lim _{r_{n} \rightarrow+\infty} \frac{\varphi\left(T\left(r_{n}, f\right)\right)}{\log r_{n}}=\rho_{\varphi}^{1}(f), \quad\left(\text { resp. } \quad \lim _{r_{n} \rightarrow+\infty} \frac{\varphi\left(e^{T\left(r_{n}, f\right)}\right)}{\log r_{n}}=\rho_{\varphi}^{0}(f)\right) .
$$

P r o o f. The definition of $\rho_{\varphi}^{1}(f)$ implies that there exists a sequence $\left\{s_{n}, n \geq 1\right\}, s_{n} \rightarrow+\infty$ such that

$$
\lim _{s_{n} \rightarrow+\infty} \frac{\varphi\left(T\left(s_{n}, f\right)\right)}{\log s_{n}}=\rho_{\varphi}^{1}(f) .
$$

Setting $m\left(E_{2}\right)=\delta<+\infty$. Then, for $r_{n} \in\left[s_{n}, s_{n}+\delta+1\right] \backslash E_{2}$, we have

$$
\frac{\varphi\left(T\left(r_{n}, f\right)\right)}{\log r_{n}} \geq \frac{\varphi\left(T\left(s_{n}, f\right)\right)}{\log \left(s_{n}+\delta+1\right)}=\frac{\varphi\left(T\left(s_{n}, f\right)\right)}{\log s_{n}+\log \left(1+\frac{\delta+1}{s_{n}}\right)} .
$$

Hence

$$
\lim _{r_{n} \rightarrow+\infty} \frac{\varphi\left(T\left(r_{n}, f\right)\right)}{\log r_{n}} \geq \lim _{s_{n} \rightarrow+\infty} \frac{\varphi\left(T\left(s_{n}, f\right)\right)}{\log s_{n}+\log \left(1+\frac{\delta+1}{s_{n}}\right)}=\rho_{\varphi}^{1}(f) .
$$

By

$$
\lim _{r_{n} \rightarrow+\infty} \frac{\varphi\left(T\left(r_{n}, f\right)\right)}{\log r_{n}} \leq \limsup _{r \rightarrow+\infty} \frac{\varphi(T(r, f))}{\log r}=\rho_{\varphi}^{1}(f)
$$

we deduce that

$$
\lim _{r_{n} \rightarrow+\infty} \frac{\varphi\left(T\left(r_{n}, f\right)\right)}{\log r_{n}}=\rho_{\varphi}^{1}(f) .
$$

Similar proof for $\rho_{\varphi}^{0}(f)$.

Lemma 7. Let $\varphi \in \Phi$ and $f$ be a meromorphic function satisfying $0<\rho_{\varphi}^{0}(f)<+\infty$ and $0<\tau_{\varphi}^{0}(f)<+\infty$. Then, for any given $\eta<\tau_{\varphi}^{0}(f)$, there exists a set $E_{3} \subset[0,+\infty)$ with infinite logarithmic measure such that for all $r \in E_{3}$, we have

$$
\varphi\left(e^{T(r, f)}\right)>\log \left(\eta r^{\rho_{\varphi}^{0}(f)}\right)
$$

P r o o f. We denote $\rho_{\varphi}^{0}(f)=\rho_{0}$ and $\tau_{\varphi}^{0}(f)=\tau_{0}$. The definition of $\tau_{\varphi}^{0}(f)$ implies that there exists a sequence $\left\{r_{m}, m \geq 1\right\}$ tending to $+\infty$ satisfying

$$
\left(1+\frac{1}{m}\right) r_{m}<r_{m+1} \quad \text { and } \quad \lim _{m \rightarrow+\infty} \frac{e^{\varphi\left(e^{T\left(r_{m}, f\right)}\right)}}{r_{m}^{\rho_{0}}}=\tau_{0} .
$$

Then, for any given $\varepsilon\left(0<\varepsilon<\tau_{0}-\eta\right)$, there exists an integer $m_{1}$ such that for all $m \geq m_{1}$, we have

$$
e^{\varphi\left(e^{T\left(r_{m}, f\right)}\right)}>\left(\tau_{0}-\varepsilon\right) r_{m}^{\rho_{0}} .
$$

Since $\eta<\tau_{0}-\varepsilon$, there exists an integer $m_{2}$ such that for all $m \geq m_{2}$, we have

$$
\left(\frac{m}{m+1}\right)^{\rho_{0}}>\frac{\eta}{\tau_{0}-\varepsilon}
$$

Taking $m \geq m_{3}=\max \left\{m_{1}, m_{2}\right\}$, it follows from (3.4) and (3.5) that for any $r \in\left[r_{m},(1+1 / m) r_{m}\right]$

$$
e^{\varphi\left(e^{T(r, f)}\right)} \geq e^{\varphi\left(e^{T\left(r_{m}, f\right)}\right)}>\left(\tau_{0}-\varepsilon\right) r_{m}^{\rho_{0}} \geq\left(\tau_{0}-\varepsilon\right)\left(\frac{m r}{m+1}\right)^{\rho_{0}}>\eta r^{\rho_{0}} .
$$


Thus

$$
\varphi\left(e^{T(r, f)}\right)>\log \left(\eta r^{\rho_{\varphi}^{0}(f)}\right)
$$

Setting $E_{3}=\bigcup_{m=m_{3}}^{+\infty}\left[r_{m},(1+1 / m) r_{m}\right]$, then the logarithmic measure $\operatorname{lm}\left(E_{3}\right)$ of $E_{3}$ satisfies

$$
\operatorname{lm}\left(E_{3}\right)=\sum_{m=m_{3}}^{+\infty} \int_{r_{m}}^{(1+1 / m) r_{m}} \frac{d t}{t}=\sum_{m=m_{3}}^{+\infty} \log \left(1+\frac{1}{m}\right)=+\infty .
$$

Lemma 8. Let $A_{0}, A_{1}, \ldots, A_{k-1}, F \not \equiv 0$ be meromorphic functions and let $f$ be a meromorphic solution of equation (1.2). If $\max \left\{\rho_{\varphi}^{1}(F), \rho_{\varphi}^{1}\left(A_{j}\right): j=0,1, \ldots, k-1\right\}<\rho_{\varphi}^{1}(f)$, then

$$
\bar{\lambda}_{\varphi}^{1}(f)=\lambda_{\varphi}^{1}(f)=\rho_{\varphi}^{1}(f) .
$$

P r o o f. Equation (1.2) can be written as

$$
\frac{1}{f}=\frac{1}{F}\left(\frac{f^{(k)}}{f}+A_{k-1} \frac{f^{(k-1)}}{f}+\cdots+A_{1} \frac{f^{\prime}}{f}+A_{0}\right) .
$$

If $f$ has a zero at $z_{0}$ of order $l>k$ and if $A_{0}, A_{1}, \ldots, A_{k-1}$ are all analytic at $z_{0}$, then $F$ has a zero at $z_{0}$ of order at least $l-k$. Then

$$
n\left(r, \frac{1}{f}\right) \leq k \cdot \bar{n}\left(r, \frac{1}{f}\right)+n\left(r, \frac{1}{F}\right)+\sum_{j=0}^{k-1} n\left(r, A_{j}\right)
$$

and

$$
N\left(r, \frac{1}{f}\right) \leq k \cdot \bar{N}\left(r, \frac{1}{f}\right)+N\left(r, \frac{1}{F}\right)+\sum_{j=0}^{k-1} N\left(r, A_{j}\right) .
$$

By the lemma of logarithmic derivative [10] and (3.6), we get that

$$
m\left(r, \frac{1}{f}\right) \leq m\left(r, \frac{1}{F}\right)+\sum_{j=0}^{k-1} m\left(r, A_{j}\right)+O(\log r+\log T(r, f))
$$

holds for all $|z|=r \notin E_{4}$, where $E_{4}$ is a set of finite linear measure. By (3.7), (3.8) and the Nevanlinna's first main theorem, we obtain

$$
\begin{gathered}
T(r, f)=T\left(r, \frac{1}{f}\right)+O(1)=m\left(r, \frac{1}{f}\right)+N\left(r, \frac{1}{f}\right)+O(1) \\
\leq k \cdot \bar{N}\left(r, \frac{1}{f}\right)+T(r, F)+\sum_{j=0}^{k-1} T\left(r, A_{j}\right)+O(\log r+\log T(r, f))
\end{gathered}
$$

holds for all sufficiently large $r \notin E_{4}$. We denote

$$
\mu=\max \left\{\rho_{\varphi}^{1}(F), \rho_{\varphi}^{1}\left(A_{j}\right)(j=0,1, \ldots, k-1)\right\} .
$$

According to Lemma 6, there exists a sequence $\left\{r_{n}, r_{n} \notin E_{4}\right\}$ such that

$$
\lim _{r_{n} \rightarrow+\infty} \frac{\varphi\left(T\left(r_{n}, f\right)\right)}{\log r_{n}}=\rho_{\varphi}^{1}(f)=\rho_{1} .
$$


So, if $r_{n} \notin E_{4}$, then for any given $\varepsilon\left(0<2 \varepsilon<\rho_{1}-\mu\right)$ we get

$$
T\left(r_{n}, f\right) \geq \varphi^{-1}\left(\left(\rho_{1}-\varepsilon\right) \log r_{n}\right) .
$$

We have

$$
\begin{gathered}
\max _{j=0,1, \ldots, k-1}\left\{T\left(r_{n}, F\right), T\left(r_{n}, A_{j}\right)\right\} \leq \varphi^{-1}\left((\mu+\varepsilon) \log r_{n}\right), \\
O\left(\log r_{n}+\log T\left(r_{n}, f\right)\right)=o\left(T\left(r_{n}, f\right)\right) .
\end{gathered}
$$

Since $\varepsilon\left(0<2 \varepsilon<\rho_{1}-\mu\right)$, then from (3.10), (3.11) and Proposition 1, we obtain

$$
\begin{aligned}
& \max _{j=0,1, \ldots, k-1}\left\{\frac{T\left(r_{n}, F\right)}{T\left(r_{n}, f\right)}, \frac{T\left(r_{n}, A_{j}\right)}{T\left(r_{n}, f\right)}\right\} \leq \frac{\exp \left\{\log \varphi^{-1}\left((\mu+\varepsilon) \log r_{n}\right)\right\}}{\exp \left\{\log \varphi^{-1}\left(\left(\rho_{1}-\varepsilon\right) \log r_{n}\right)\right\}} \\
& =\exp \left\{\log \varphi^{-1}\left((\mu+\varepsilon) \log r_{n}\right)-\log \varphi^{-1}\left(\left(\rho_{1}-\varepsilon\right) \log r_{n}\right)\right\} \\
& =\exp \left\{\left(1-\frac{\log \varphi^{-1}\left(\left(\rho_{1}-\varepsilon\right) \log r_{n}\right)}{\log \varphi^{-1}\left((\mu+\varepsilon) \log r_{n}\right)}\right) \log \varphi^{-1}\left((\mu+\varepsilon) \log r_{n}\right)\right\} \longrightarrow 0
\end{aligned}
$$

as $r_{n} \rightarrow+\infty$. By substituting (3.12) and (3.13) into (3.9) we deduce that for sufficiently large $r_{n} \notin E_{4}$, there holds

$$
(1-o(1)) T\left(r_{n}, f\right) \leq k \bar{N}\left(r_{n}, \frac{1}{f}\right) .
$$

From this inequality, by the monotonicity of $\varphi$ and (3.3), we obtain $\rho_{\varphi}^{1}(f) \leq \bar{\lambda}_{\varphi}^{1}(f)$. In addition, we have by definition that $\bar{\lambda}_{\varphi}^{1}(f) \leq \lambda_{\varphi}^{1}(f) \leq \rho_{\varphi}^{1}(f)$. Hence $\bar{\lambda}_{\varphi}^{1}(f)=\lambda_{\varphi}^{1}(f)=\rho_{\varphi}^{1}(f)$.

Lemma 9. Let $f$ be a meromorphic function. If $\rho_{\varphi}^{0}(f)=\rho<+\infty$, then $\rho_{\varphi}^{1}(f)=0$.

P r o o f. Suppose that $\rho_{\varphi}^{0}(f)=\rho<+\infty$. Then, for any given $\varepsilon>0$ and sufficiently large $r$, we have

$$
T(r, f) \leq \log \left(\varphi^{-1}((\rho+\varepsilon) \log r)\right) .
$$

By Karamata's theorem (see [19]), it follows that $\varphi\left(e^{t}\right)=t^{o(1)}$ as $t \rightarrow+\infty$. Hence,

$$
\begin{gathered}
\rho_{\varphi}^{1}(f)=\limsup _{r \rightarrow+\infty} \frac{\varphi(T(r, f))}{\log r}=\limsup _{r \rightarrow+\infty} \frac{\varphi\left(e^{\log T(r, f)}\right)}{\log r} \\
=\limsup _{r \rightarrow+\infty} \frac{(\log T(r, f))^{o(1)}}{\log r} \leq \limsup _{r \rightarrow+\infty} \frac{\left(\log \log \left(\varphi^{-1}((\rho+\varepsilon) \log r)\right)\right)^{o(1)}}{\log r}=0 .
\end{gathered}
$$

\section{Proofs of the main results}

Proof of Theorem 1. (i) We first prove that $\rho_{\varphi}^{1}(f) \leq \rho_{\varphi}^{0}\left(A_{s}\right) \leq \rho_{\varphi}^{0}(f)$ holds for every transcendental meromorphic function satisfying (1.1). From equation (1.1), we know that the poles of $f$ can only occur at the poles of $A_{0}, A_{1}, \ldots, A_{k-1}$, note that the multiplicities of poles of $f$ are uniformly bounded, so we have

$$
N(r, f) \leq C_{1} \bar{N}(r, f) \leq C_{1} \sum_{j=0}^{k-1} \bar{N}\left(r, A_{j}\right) \leq C \max \left\{N\left(r, A_{j}\right): j=0,1, \ldots, k-1\right\} \leq O\left(T\left(r, A_{s}\right)\right),
$$


where $C$ and $C_{1}$ are two suitable positive constants. Hence

$$
T(r, f) \leq m(r, f)+O\left(T\left(r, A_{s}\right)\right) .
$$

This inequality and Lemma 1 lead to

$$
T(r, f) \leq m(r, f)+O\left(T\left(r, A_{s}\right)\right) \leq O\left(e^{T\left(r, A_{s}\right)\left[(\log r) \log T\left(r, A_{s}\right)\right]^{\gamma}}\right), \quad \gamma>1
$$

outside of an exceptional set $E_{0}$ with finite logarithmic measure. By the monotonicity of the function $\varphi$ and (3.3), we obtain $\rho_{\varphi}^{1}(f) \leq \rho_{\varphi}^{0}\left(A_{s}\right)$.

On the other hand, equation (1.1) can be written as

$$
\begin{aligned}
& -A_{s}=\frac{f^{(k)}}{f^{(s)}}+A_{k-1} \frac{f^{(k-1)}}{f^{(s)}}+\cdots+A_{s+1} \frac{f^{(s+1)}}{f^{(s)}}+A_{s-1} \frac{f^{(s-1)}}{f^{(s)}}+\cdots+A_{0} \frac{f}{f^{(s)}} \\
& =\frac{f}{f^{(s)}}\left(\frac{f^{(k)}}{f}+A_{k-1} \frac{f^{(k-1)}}{f}+\cdots+A_{s+1} \frac{f^{(s+1)}}{f}+A_{s-1} \frac{f^{(s-1)}}{f}+\cdots+A_{0}\right) .
\end{aligned}
$$

By the lemma of logarithmic derivative and the fact that

$$
m\left(r, \frac{f}{f^{(s)}}\right) \leq T(r, f)+T\left(r, \frac{1}{f^{(s)}}\right)=T(r, f)+T\left(r, f^{(s)}\right)+O(1)=O(T(r, f)),
$$

it follows that

$$
T\left(r, A_{s}\right) \leq N\left(r, A_{s}\right)+\sum_{j \neq s} m\left(r, A_{j}\right)+O(\log r+\log T(r, f))+O(T(r, f))
$$

which holds for all $|z|=r \notin E_{5}$ where $E_{5}$ is a set of finite linear measure. By Lemma 6 , it follows that there exists a sequence $\left\{r_{n}, n \geq 1\right\}, r_{n} \rightarrow+\infty$ such that for $\left|z_{n}\right|=r_{n} \notin E_{5}$

$$
\lim _{r_{n} \rightarrow+\infty} \frac{\varphi\left(e^{T\left(r_{n}, A_{s}\right)}\right)}{\log r_{n}}=\rho_{\varphi}^{0}\left(A_{s}\right)=\rho_{0}
$$

and so

$$
T\left(r_{n}, A_{s}\right) \geq \log \left(\varphi^{-1}\left(\left(\rho_{0}-\varepsilon\right) \log r_{n}\right)\right) .
$$

Under the assumption $\eta=\max \left\{\rho_{\varphi}^{0}\left(A_{j}\right), \lambda_{\varphi}^{0}\left(1 / A_{s}\right): j \neq s\right\}<\rho_{\varphi}^{0}\left(A_{s}\right)=\rho_{0}$, we have

$$
\begin{gathered}
N\left(r_{n}, A_{s}\right) \leq \log \left(\varphi^{-1}\left((\eta+\varepsilon) \log r_{n}\right)\right), \\
m\left(r_{n}, A_{j}\right) \leq T\left(r_{n}, A_{j}\right) \leq \log \left(\varphi^{-1}\left((\eta+\varepsilon) \log r_{n}\right)\right), j \neq s
\end{gathered}
$$

provided for any given $\varepsilon$ that verifies $0<2 \varepsilon<\rho_{0}-\eta$. Substituting (4.2), (4.3) and (4.4) into (4.1), we get

$$
(1-o(1)) \log \left(\varphi^{-1}\left(\left(\rho_{0}-\varepsilon\right) \log r_{n}\right)\right) \leq O\left(\log r_{n}+\log T\left(r_{n}, f\right)\right)+O\left(T\left(r_{n}, f\right)\right)=O\left(T\left(r_{n}, f\right)\right) .
$$

Applying (3.3), one can deduce that $\rho_{\varphi}^{0}\left(A_{s}\right)=\rho_{0} \leq \rho_{\varphi}^{0}(f)$.

(ii) Now, we prove that there exists at least one meromorphic solution that satisfies $\rho_{\varphi}^{1}(f)=\rho_{\varphi}^{0}\left(A_{s}\right)$. Let $\left\{f_{1}, f_{2}, \ldots, f_{k}\right\}$ be a solution base of equation (1.1). By Lemma 2, we have

$$
e^{m\left(r, A_{s}\right)} \leq O\left(\max _{1 \leq i \leq k} T\left(r, f_{i}\right)\right), \quad s \in\{1,2, \ldots, k-1\} .
$$


If $N\left(r, A_{s}\right) \geq m\left(r, A_{s}\right)$, so $T\left(r, A_{s}\right) \leq 2 N\left(r, A_{s}\right)$, then $\rho_{\varphi}^{0}\left(A_{s}\right) \leq \lambda_{\varphi}^{0}\left(\frac{1}{A_{s}}\right)$. This contradicts our assumption $\lambda_{\varphi}^{0}\left(\frac{1}{A_{s}}\right)<\rho_{\varphi}^{0}\left(A_{s}\right)$ and asserts that $N\left(r, A_{s}\right)<m\left(r, A_{s}\right)$. Hence, for sufficiently large $r$, we have

$$
e^{T\left(r, A_{s}\right)}=O\left(e^{m\left(r, A_{s}\right)}\right) \leq O\left(\max _{1 \leq i \leq k} T\left(r, f_{i}\right)\right) .
$$

This implies that there exists at least one solution of $\left\{f_{1}, f_{2}, \ldots, f_{k}\right\}$, say $f_{1}$, that satisfies $e^{T\left(r, A_{s}\right)} \leq O\left(T\left(r, f_{1}\right)\right)$. By this inequality and (3.3) and the monotonicity of $\varphi$, we obtain

$$
\rho_{\varphi}^{0}\left(A_{s}\right) \leq \rho_{\varphi}^{1}\left(f_{1}\right)
$$

We have proved in the first part that $\rho_{\varphi}^{1}\left(f_{1}\right) \leq \rho_{\varphi}^{0}\left(A_{s}\right)$. Therefore, $\rho_{\varphi}^{1}\left(f_{1}\right)=\rho_{\varphi}^{0}\left(A_{s}\right)$.

P r o o f of Theorem 2. Assume that $f$ is a non-zero meromorphic solution whose poles are of uniformly bounded multiplicities of (1.1). Equation (1.1) can be written as

$$
A_{0}=-\left(\frac{f^{(k)}}{f}+A_{k-1} \frac{f^{(k-1)}}{f}+\cdots+A_{1} \frac{f^{\prime}}{f}\right) .
$$

By the lemma of logarithmic derivative and the above equation, we have

$$
\begin{gathered}
m\left(r, A_{0}\right) \leq \sum_{j=1}^{k-1} m\left(r, A_{j}\right)+\sum_{j=1}^{k} m\left(r, \frac{f^{(j)}}{f}\right)+O(1) \\
\leq \sum_{j=1}^{k-1} m\left(r, A_{j}\right)+O(\log r+\log T(r, f))
\end{gathered}
$$

holds possibly outside of an exceptional set $E_{6} \subset(0,+\infty)$ with finite linear measure. From this inequality, it follows

$$
\begin{gathered}
T\left(r, A_{0}\right)=m\left(r, A_{0}\right)+N\left(r, A_{0}\right) \\
\leq N\left(r, A_{0}\right)+\sum_{j=1}^{k-1} m\left(r, A_{j}\right)+O(\log r+\log T(r, f))
\end{gathered}
$$

holds for $r \notin E_{6}$. By Lemma 6 , it follows that there exists a sequence $\left\{r_{n}, n \geq 1\right\}, r_{n} \rightarrow+\infty$ such that for $\left|z_{n}\right|=r_{n} \notin E_{6}$

$$
\lim _{r_{n} \rightarrow+\infty} \frac{\varphi\left(e^{T\left(r_{n}, A_{0}\right)}\right)}{\log r_{n}}=\rho_{\varphi}^{0}\left(A_{0}\right)=\rho_{0}
$$

and so

$$
T\left(r_{n}, A_{0}\right) \geq \log \left(\varphi^{-1}\left(\left(\rho_{0}-\varepsilon\right) \log r_{n}\right)\right)
$$

under the assumption $\eta=\max \left\{\rho_{\varphi}^{0}\left(A_{j}\right), \lambda_{\varphi}^{0}\left(1 / A_{0}\right): j \neq 0\right\}<\rho_{\varphi}^{0}\left(A_{0}\right)=\rho_{0}$, we have

$$
\begin{gathered}
N\left(r_{n}, A_{0}\right) \leq \log \left(\varphi^{-1}\left((\eta+\varepsilon) \log r_{n}\right)\right), \\
m\left(r_{n}, A_{j}\right) \leq T\left(r_{n}, A_{j}\right) \leq \log \left(\varphi^{-1}\left((\eta+\varepsilon) \log r_{n}\right)\right), \quad j \neq 0
\end{gathered}
$$

provided for any given $\varepsilon$ that verifies $0<2 \varepsilon<\rho_{0}-\eta$. Substituting (4.7), (4.8) and (4.9) into (4.6), we get

$$
(1-o(1)) \log \left(\varphi^{-1}\left(\left(\rho_{0}-\varepsilon\right) \log r_{n}\right)\right) \leq O\left(\log r_{n}+\log T\left(r_{n}, f\right)\right) .
$$


Applying (3.3), one can deduce that $\rho_{\varphi}^{0}\left(A_{0}\right)=\rho_{0} \leq \rho_{\varphi}^{1}(f)$.

On the other hand, from Theorem 1 , we have $\rho_{\varphi}^{0}\left(A_{0}\right) \geq \rho_{\varphi}^{1}(f)$. We deduce finally that every meromorphic solution $f \not \equiv 0$ whose poles are of uniformly bounded multiplicities of (1.1) satisfies $\rho_{\varphi}^{1}(f)=\rho_{\varphi}^{0}\left(A_{0}\right)$.

Proof of Theorem 3. Assume that $f$ is a non-zero meromorphic solution whose poles are of uniformly bounded multiplicities of (1.1). If $\lambda_{\varphi}^{0}\left(1 / A_{0}\right)<\rho_{\varphi}^{0}\left(A_{0}\right)$ and

$$
\max \left\{\rho_{\varphi}^{0}\left(A_{j}\right): j=1, \ldots, k-1\right\}<\rho_{\varphi}^{0}\left(A_{0}\right)<+\infty,
$$

then by Theorem 2, we obtain $\rho_{\varphi}^{1}(f)=\rho_{\varphi}^{0}\left(A_{0}\right)$. Suppose that $\lambda_{\varphi}^{0}\left(1 / A_{0}\right)<\rho_{\varphi}^{0}\left(A_{0}\right)$ and

$$
\begin{gathered}
\max \left\{\rho_{\varphi}^{0}\left(A_{j}\right): j=1, \ldots, k-1\right\}=\rho_{\varphi}^{0}\left(A_{0}\right)=\rho_{0}\left(0<\rho_{0}<+\infty\right), \\
\max \left\{\tau_{\varphi}^{0}\left(A_{j}\right): \rho_{\varphi}^{0}\left(A_{j}\right)=\rho_{\varphi}^{0}\left(A_{0}\right)\right\}<\tau_{\varphi}^{0}\left(A_{0}\right)=\tau_{0}\left(0<\tau_{0}<+\infty\right) .
\end{gathered}
$$

Then, there exists a set $J \subseteq\{1, \ldots, k-1\}$ such that $\rho_{\varphi}^{0}\left(A_{j}\right)=\rho_{\varphi}^{0}\left(A_{0}\right)=\rho_{0}(j \in J)$ and $\tau_{\varphi}^{0}\left(A_{j}\right)<$ $\tau_{\varphi}^{0}\left(A_{0}\right)=\tau_{0}(j \in J)$. Hence, there exist two constants $\beta_{1}$ and $\beta_{2}$ such that

$$
\max \left\{\tau_{\varphi}^{0}\left(A_{j}\right): j \in J\right\}<\beta_{1}<\beta_{2}<\tau_{\varphi}^{0}\left(A_{0}\right)=\tau_{0} .
$$

The definition of the type $\tau_{\varphi}^{0}\left(A_{j}\right)$ implies that for $r$ sufficiently large

$$
e^{m\left(r, A_{j}\right)} \leq e^{T\left(r, A_{j}\right)}<\varphi^{-1}\left(\log \left(\beta_{1} r^{\rho_{0}}\right)\right), \quad j \in J
$$

and

$$
e^{m\left(r, A_{j}\right)} \leq e^{T\left(r, A_{j}\right)}<\varphi^{-1}\left(\log \left(r^{\rho_{0}^{0}}\right)\right)<\varphi^{-1}\left(\log \left(\beta_{1} r^{\rho_{0}}\right)\right), \quad j \in\{1, \ldots, k-1\} \backslash J,
$$

where $0<\rho_{0}^{0}<\rho_{0}$. Since $\lambda_{0}=\lambda_{\varphi}^{0}\left(1 / A_{0}\right)<\rho_{\varphi}^{0}\left(A_{0}\right)=\rho_{0}$, then for any given $\varepsilon\left(0<2 \varepsilon<\rho_{0}-\lambda_{0}\right)$ and sufficiently large $r$, we have

$$
e^{N\left(r, A_{0}\right)} \leq \varphi^{-1}\left(\log \left(r^{\lambda_{0}+\varepsilon}\right)\right)<\varphi^{-1}\left(\log \left(r^{\rho_{0}-\varepsilon}\right)\right)<\varphi^{-1}\left(\log \left(\beta_{1} r^{\rho_{0}}\right)\right) .
$$

By Lemma 7 , there exists a set $E_{3} \subset[1,+\infty)$ with infinite logarithmic measure such that for all $r \in E_{3}$, we have

$$
e^{T\left(r, A_{0}\right)}>\varphi^{-1}\left(\log \left(\beta_{2} r^{\rho_{0}}\right)\right) .
$$

By substituting (4.10), (4.11), (4.12) and (4.13) into (4.6), we obtain

$$
(1-o(1)) \log \left(\varphi^{-1}\left[\log \left(\beta_{2} r^{\rho_{0}}\right)\right]\right) \leq O(\log r+\log T(r, f))
$$

for all $r \in E_{3} \backslash E_{6}$. Since $E_{3} \backslash E_{6}$ is a set of infinite logarithmic measure, then there exists a sequence of points $\left|z_{n}\right|=r_{n} \in E_{3} \backslash E_{6}$ tending to $+\infty$. Hence, by (4.14) we have

$$
(1-o(1)) \log \left(\varphi^{-1}\left[\log \left(\beta_{2} r_{n}^{\rho_{0}}\right)\right]\right) \leq O\left(\log r_{n}+\log T\left(r_{n}, f\right)\right)
$$

holds for all $z_{n}$ satisfying $\left|z_{n}\right|=r_{n} \in E_{3} \backslash E_{6}$ as $\left|z_{n}\right|=r_{n} \rightarrow+\infty$. By the monotonicity of $\varphi^{-1}$ and (3.3), we obtain $\rho_{\varphi}^{0}\left(A_{0}\right) \leq \rho_{\varphi}^{1}(f)$. By Theorem 1 , we have $\rho_{\varphi}^{1}(f) \leq \rho_{\varphi}^{0}\left(A_{0}\right)$. Therefore $\rho_{\varphi}^{1}(f)=\rho_{\varphi}^{0}\left(A_{0}\right)$ which completes the proof.

P r oof of Theorem 4. Since all solutions of equation (1.2) are meromorphic functions, all solutions of the homogeneous differential equation (1.1) corresponding to equation (1.2) are also 
meromorphic functions. We assume that $\left\{f_{1}, \ldots, f_{k}\right\}$ is a meromorphic solution base of (1.1), then any solution of (1.2) has the form

$$
f=c_{1} f_{1}+c_{2} f_{2}+\cdots+c_{k} f_{k},
$$

where $c_{1}, c_{2}, \ldots, c_{k}$ are meromorphic functions satisfying

$$
c_{j}^{\prime}=F \cdot G_{j}\left(f_{1}, \ldots, f_{k}\right) \cdot W^{-1}\left(f_{1}, \ldots, f_{k}\right), \quad j=1,2, \ldots, k,
$$

where $G_{j}\left(f_{1}, \ldots, f_{k}\right)$ are differential polynomials in $\left\{f_{1}, \ldots, f_{k}\right\}$ and their derivatives and $W^{-1}\left(f_{1}, \ldots, f_{k}\right)$ is the Wronskian of $\left\{f_{1}, \ldots, f_{k}\right\}$. We have by Theorem 2

$$
\rho_{\varphi}^{1}\left(f_{j}\right)=\rho_{\varphi}^{0}\left(A_{0}\right), \quad j=1, \ldots, k .
$$

By Lemma 4, Lemma 5, (4.15) and (4.16), we get

$$
\rho_{\varphi}^{1}(f) \leq \max \left\{\rho_{\varphi}^{1}\left(f_{j}\right)(j=1, \ldots, k), \rho_{\varphi}^{1}(F)\right\}=\rho_{\varphi}^{0}\left(A_{0}\right) .
$$

In order to show that all solutions $f$ of equation $(1.2)$ satisfy $\rho_{\varphi}^{1}(f)=\rho_{\varphi}^{0}\left(A_{0}\right)$ with at most one exceptional solution, say $f_{1}$, satisfying $\rho_{\varphi}^{1}\left(f_{1}\right)<\rho_{\varphi}^{0}\left(A_{0}\right)$, we suppose that there exist two distinct meromorphic solutions $f_{1}$ and $f_{2}$ of equation (1.2) satisfying $\rho_{\varphi}^{1}\left(f_{i}\right)<\rho_{\varphi}^{0}\left(A_{0}\right), i=1,2$. Then, $f=f_{1}-f_{2}$ is also a non-zero meromorphic solution of (1.1) and satisfies

$$
\rho_{\varphi}^{1}(f)=\rho_{\varphi}^{1}\left(f_{1}-f_{2}\right) \leq \max \left\{\rho_{\varphi}^{1}\left(f_{1}\right), \rho_{\varphi}^{1}\left(f_{2}\right)\right\}<\rho_{\varphi}^{0}\left(A_{0}\right)
$$

which contradicts Theorem 2. By (2.3) for all solutions $f$ of equation (1.2) satisfying $\rho_{\varphi}^{1}(f)=$ $\rho_{\varphi}^{0}\left(A_{0}\right)$, by Lemma 9 , we have

$$
\max \left\{\rho_{\varphi}^{1}(F), \rho_{\varphi}^{1}\left(A_{j}\right)(j=0,1, \ldots, k-1)\right\}=\rho_{\varphi}^{1}(F)<\rho_{\varphi}^{0}\left(A_{0}\right)=\rho_{\varphi}^{1}(f) .
$$

By Lemma 8, we have $\bar{\lambda}_{\varphi}^{1}(f)=\lambda_{\varphi}^{1}(f)=\rho_{\varphi}^{1}(f)$ and hence Theorem 4 is proved.

P r oof of Theorem 5. Let $f$ be a meromorphic solution of equation (1.2) and $\left\{f_{1}, \ldots, f_{k}\right\}$ be a meromorphic solution base of (1.1) corresponding to equation (1.2). By a similar discussion as in the proof of Theorem 4, it follows from Lemma 4, Lemma 5, (4.15) and (4.16) that

$$
\rho_{\varphi}^{1}(f) \leq \max \left\{\rho_{\varphi}^{1}\left(f_{j}\right)(j=1, \ldots, k), \rho_{\varphi}^{1}(F)\right\} .
$$

By the first part of the proof of Theorem 1, one can show easily that

$$
\rho_{\varphi}^{1}\left(f_{j}\right) \leq \max \left\{\rho_{\varphi}^{0}\left(A_{j}\right): j=0, \ldots, k-1\right\}
$$

for $j=1, \ldots, k$. We obtain from the assumptions of Theorem 5 that $\rho_{\varphi}^{1}\left(f_{j}\right) \leq \rho_{\varphi}^{1}(F)$ and thus

$$
\rho_{\varphi}^{1}(f) \leq \rho_{\varphi}^{1}(F) .
$$

On the other hand, by Lemma 4, Lemma 5 and a simple order comparison from equation (1.2), we get

$$
\rho_{\varphi}^{1}(F) \leq \max \left\{\rho_{\varphi}^{1}\left(A_{j}\right)(j=0, \ldots, k-1), \rho_{\varphi}^{1}(f)\right\} .
$$

Since $\rho_{\varphi}^{1}\left(A_{j}\right) \leq \rho_{\varphi}^{0}\left(A_{j}\right)<\rho_{\varphi}^{1}(F)(j=0, \ldots, k-1)$, then

$$
\rho_{\varphi}^{1}(F) \leq \rho_{\varphi}^{1}(f) .
$$


Therefore, $\rho_{\varphi}^{1}(f)=\rho_{\varphi}^{1}(F)$.

Proof of Theorem 6. Assume that $f$ is a non-zero meromorphic solution whose poles are of uniformly bounded multiplicities of (1.1). Set $G_{1}=\{|z|=r: z \in G\}$, since $\overline{\log \operatorname{dens}}\{|z|: z \in$ $G\}>0$, then $G_{1}$ is a set with $\int_{G_{1}} \frac{d r}{r}=+\infty$. Set

$$
\delta\left(\infty, A_{0}\right)=\liminf _{r \rightarrow+\infty} \frac{m\left(r, A_{0}\right)}{T\left(r, A_{0}\right)}=\delta>0 .
$$

Thus, for sufficiently large $r$, we have

$$
m\left(r, A_{0}\right)>\frac{1}{2} \delta T\left(r, A_{0}\right) .
$$

By substituting (2.4), (2.5) and (4.19) into (4.5), we obtain for sufficiently large $r$ and any given $\varepsilon$ $(0<2 \varepsilon<\alpha-\beta)$

$$
\begin{gathered}
\frac{1}{2} \delta \log \left(\varphi^{-1}((\alpha-\varepsilon) \log r)\right) \leq \frac{1}{2} \delta T\left(r, A_{0}\right) \leq m\left(r, A_{0}\right) \\
\leq \sum_{j=1}^{k-1} m\left(r, A_{j}\right)+\sum_{j=1}^{k} m\left(r, \frac{f^{(j)}}{f}\right)+O(1) \\
\leq \sum_{j=1}^{k-1} T\left(r, A_{j}\right)+O(\log r+\log T(r, f)) \\
\leq(k-1) \log \left(\varphi^{-1}(\beta \log r)\right)+O(\log r+\log T(r, f)),
\end{gathered}
$$

it follows that

$$
(1-o(1)) \log \left(\varphi^{-1}((\alpha-\varepsilon) \log r)\right) \leq O(\log r+\log T(r, f))
$$

holds for all $z$ satisfying $|z|=r \in G_{1} \backslash E_{6}$ as $|z|=r \rightarrow+\infty$. Since $G_{1} \backslash E_{6}$ is a set of infinite logarithmic measure, then there exists a sequence of points $\left|z_{n}\right|=r_{n} \in G_{1} \backslash E_{6}$ tending to $+\infty$. Hence, by (4.20) we have

$$
(1-o(1)) \log \left(\varphi^{-1}\left((\alpha-\varepsilon) \log r_{n}\right)\right) \leq O\left(\log r_{n}+\log T\left(r_{n}, f\right)\right)
$$

holds for all $z_{n}$ satisfying $\left|z_{n}\right|=r_{n} \in G_{1} \backslash E_{6}$ as $\left|z_{n}\right|=r_{n} \rightarrow+\infty$. By the monotonicity of $\varphi^{-1}$ and arbitrariness of $\varepsilon(0<2 \varepsilon<\alpha-\beta)$, one can obtain $\rho_{\varphi}^{1}(f) \geq \alpha$.

On the other hand, it follows by a similar proof as in the first part of Theorem 1 that $\rho_{\varphi}^{1}(f) \leq \alpha$. Therefore $\rho_{\varphi}^{1}(f)=\alpha$.

P r o of of Theorem 7. (i) If $\rho_{\varphi}^{1}(F) \geq \alpha$, then it follows from Theorem 5 that $\rho_{\varphi}^{1}(f)=\rho_{\varphi}^{1}(F)$.

(ii) If $\rho_{\varphi}^{1}(F)<\alpha$, we prove that $\rho_{1}=\rho_{\varphi}^{1}(f)=\alpha$ for any non-zero meromorphic solution whose poles are of uniformly bounded multiplicities of (1.1). We show firstly that $\rho_{1}=\rho_{\varphi}^{1}(f) \geq \alpha$. Without loss of the generality, we suppose the contrary $\rho_{1} \leq \beta<\alpha$. Set $G_{2}=\{|z|=r: z \in G\}$, since $\overline{\log \operatorname{dens}}\{|z|: z \in G\}>0$, then $G_{2}$ is a set with $\int_{G_{2}} \frac{d r}{r}=+\infty$. From Lemma 3 , there exists a set $E_{1} \subset(1,+\infty)$ with finite logarithmic measure and a constant $B>0$ such that for all $z$ satisfying $|z|=r \notin[0,1] \cup E_{1}$, we have

$$
\left|\frac{f^{(j)}(z)}{f(z)}\right| \leq B[T(2 r, f)]^{k+1}, \quad j=1, \ldots, k .
$$


If $f$ is a non-zero meromorphic solution of equation (1.1), then

$$
\left|A_{0}(z)\right| \leq\left|\frac{f^{(k)}(z)}{f(z)}\right|+\left|A_{k-1}(z)\right|\left|\frac{f^{(k-1)}(z)}{f(z)}\right|+\cdots+\left|A_{1}(z)\right|\left|\frac{f^{\prime}(z)}{f(z)}\right| .
$$

By the definition of $\rho_{1}=\rho_{\varphi}^{1}(f)$ and substituting (2.6), (2.7), (4.21) into (4.22), we obtain

$$
\begin{gathered}
\varphi^{-1}((\alpha-\varepsilon) \log r) \leq\left|A_{0}(z)\right| \leq k B \varphi^{-1}(\beta \log r)[T(2 r, f)]^{k+1} \\
\leq k B \varphi^{-1}(\beta \log r)\left[\varphi^{-1}\left(\left(\rho_{1}+\frac{\varepsilon}{2}\right) \log 2 r\right)\right]^{k+1} \\
\leq\left[\varphi^{-1}\left(\left(\beta+\frac{\varepsilon}{2}\right) \log 2 r\right)\right]^{k+2} \leq \varphi^{-1}((\beta+\varepsilon) \log r)
\end{gathered}
$$

holds for all $z$ satisfying $|z|=r \in G_{2} \backslash\left([0,1] \cup E_{1}\right)$ as $|z|=r \rightarrow+\infty$. Since $G_{2} \backslash E_{1}$ is a set of infinite logarithmic measure, then there exists a sequence of points $\left|z_{n}\right|=r_{n} \in G_{2} \backslash E_{1}$ tending to $+\infty$. Hence, by $(4.23)$ we have

$$
\varphi^{-1}\left((\alpha-\varepsilon) \log r_{n}\right) \leq \varphi^{-1}\left((\beta+\varepsilon) \log r_{n}\right)
$$

holds for all $z_{n}$ satisfying $\left|z_{n}\right|=r_{n} \in G_{2} \backslash E_{1}$ as $\left|z_{n}\right|=r_{n} \rightarrow+\infty$. By the monotonicity of $\varphi^{-1}$ and arbitrariness of $\varepsilon(0<2 \varepsilon<\alpha-\beta)$, one can see that $\alpha \leq \beta$ which contradicts our assumption. Then, $\rho_{\varphi}^{1}(f) \geq \alpha$.

On the other hand, it follows by a similar proof in Theorem 1 that

$$
\rho_{\varphi}^{1}(f) \leq \alpha
$$

Therefore $\rho_{\varphi}^{1}(f)=\alpha$. In order to show that all solutions $f$ of equation (1.2) satisfy $\rho_{\varphi}^{1}(f)=\alpha$ with at most one exceptional solution, say $f_{0}$, satisfying $\rho_{\varphi}^{1}\left(f_{0}\right)<\alpha$, we suppose that there exist two distinct meromorphic solutions $f_{0}$ and $f_{0}^{*}$ of equation (1.2) satisfying $\max \left\{\rho_{\varphi}^{1}\left(f_{0}\right), \rho_{\varphi}^{1}\left(f_{0}^{*}\right)\right\}<\alpha$. Then, $f=f_{0}-f_{0}^{*}$ is also a non-zero meromorphic solution of (1.1) and satisfies

$$
\rho_{\varphi}^{1}(f)=\rho_{\varphi}^{1}\left(f_{0}-f_{0}^{*}\right) \leq \max \left\{\rho_{\varphi}^{1}\left(f_{0}\right), \rho_{\varphi}^{1}\left(f_{0}^{*}\right)\right\}<\alpha
$$

which contradicts the proof of the first part of (ii). By assumptions of Theorem 7, for all solutions $f$ of equation (1.2) satisfying $\rho_{\varphi}^{1}(f)=\alpha$, we have by Lemma 9

$$
\max \left\{\rho_{\varphi}^{1}(F), \rho_{\varphi}^{1}\left(A_{j}\right), j=0,1, \ldots, k-1\right\}=\rho_{\varphi}^{1}(F)<\alpha=\rho_{\varphi}^{1}(f) .
$$

By using Lemma 8, we obtain $\bar{\lambda}_{\varphi}^{1}(f)=\lambda_{\varphi}^{1}(f)=\rho_{\varphi}^{1}(f)$ and hence

$$
\bar{\lambda}_{\varphi}^{1}(f)=\lambda_{\varphi}^{1}(f)=\rho_{\varphi}^{1}(f)=\alpha
$$

with at most one exceptional solution $f_{0}$ satisfying $\rho_{\varphi}^{1}\left(f_{0}\right)<\alpha$.

\section{Conclusion}

In this paper, by using the concepts of $\varphi$-order and $\varphi$-type, we have studied the growth of meromorphic solutions of higher order linear differential equations when among meromorphic coefficients having the maximal $\varphi$-order, exactly one has its $\varphi$-type stricly greater than others. Many previous results due to Chyzhykov-Semochko, Belaïdi, Cao-Xu-Chen, Kinnunen have been extended. Now, it is interesting to study the growth of meromorphic solutions of such equations by using the concept of $(\alpha, \beta)$-order called the generalized order introduced by Sheremeta [20], see the recent paper of Mulyava-Sheremeta-Trukhan [17]. 


\section{Acknowledgements}

The authors are grateful to the referees for their many valuable remarks and suggestions which lead to the improvement of the original version of this paper.

This work was supported by the Directorate-General for Scientific Research and Technological Development (DGRSDT).

\section{REFERENCES}

1. Belaïdi B. Fast growing solutions to linear differential equations with entire coefficients having the same $\rho_{\varphi}$-order. J. Math. Appl., 2019. Vol. 42. P. 63-77. DOI: 10.7862/rf.2019.4

2. Belaïdi B. Growth of $\rho_{\varphi}$-order solutions of linear differential equations with entire coefficients. PanAmerican Math. J., 2017. Vol. 27, No. 4. P. 26-42. URL: http://www.internationalpubls.com

3. Belaïdi B. Growth and oscillation of solutions to linear differential equations with entire coefficients having the same order. Electron. J. Differential Equations, 2009. No. 70. P. 1-10. URL: https://ejde.math.txstate.edu

4. Bernal L. G. On growth $k$-order of solutions of a complex homogeneous linear differential equation. Proc. Amer. Math. Soc., 1987. Vol. 101, No. 2. P. 317-322. DOI: 10.1090/S0002-9939-1987-0902549-5

5. Cao T.-B., Xu J.F., Chen Z.X. On the meromorphic solutions of linear differential equations on the complex plane. J. Math. Anal. Appl., 2010. Vol. 364, No. 1. P. 130-142. DOI: 10.1016/j.jmaa.2009.11.018

6. Chiang Y.-M., Hayman W.K. Estimates on the growth of meromorphic solutions of linear differential equations. Comment. Math. Helv., 2004. Vol. 79, No. 3. P. 451-470. DOI: 10.1007/s00014-003-0792-7

7. Chyzhykov I., Semochko N. Fast growing entire solutions of linear differential equations. Math. Bull. Shevchenko Sci. Soc., 2016. Vol. 13. P. 68-83.

8. Frank G., Hellerstein S. On the meromorphic solutions of non-homogeneous linear differential equations with polynomial coefficients. Proc. London Math. Soc., 1986. Vol. s3-53, No. 3. P. 407-428. DOI: $10.1112 / \mathrm{plms} / \mathrm{s} 3-53.3 .407$

9. Gundersen G. G. Estimates for the logarithmic derivative of a meromorphic function, plus similar estimates. J. London Math. Soc., 1988. Vol. s2-37, No. 1. P. 88-104. DOI: 10.1112/jlms/s2-37.121.88

10. Hayman W. K. Meromorphic Functions. Oxford: Oxford Mathematical Monographs Clarendon Press, 1964. $191 \mathrm{p}$.

11. Juneja O. P., Kapoor G. P., Bajpai S. K. On the $(p, q)$-order and lower $(p, q)$-order of an entire function. J. Reine Angew. Math., 1976. Vol. 282. P. 53-67. DOI: 10.1515/crll.1976.282.53

12. Kara M.A., Belaïdi B. Some estimates of the $\varphi$-order and the $\varphi$-type of entire and meromorphic functions. Int. J. Open Problems Complex Analysis, 2019. Vol. 10, No. 3. P. 42-58. URL: http://www.i-csrs.org

13. Kinnunen L. Linear differential equations with solutions of finite iterated order. Southeast Asian Bull. Math. 1998. Vol. 22, No. 4. P. 385-405.

14. Laine I. Nevanlinna Theory and Complex Differential Equations. De Gruyter Studies in Mathematics, Vol. 15. Berlin: Walter de Gruyter \& Co., 1993. 341 p. DOI: 10.1515/9783110863147

15. Li L.M., Cao T.B. Solutions for linear differential equations with meromorphic coefficients of $[p, q]$-order in the plane. Electron. J. Differential Equations, 2012. No. 195. P. 1-15. URL: https://ejde.math.txstate.edu

16. Liu J., Tu J., Shi L. Z. Linear differential equations with entire coefficients of $[p, q]$-order in the complex plane. J. Math. Anal. Appl., 2010. Vol. 372. P. 55-67. DOI: 10.1016/j.jmaa.2010.05.014

17. Mulyava O.M., Sheremeta M.M., Trukhan Yu.S. Properties of solutions of a heterogeneous differential equation of the second order. Carpathian Math. Publ., 2019. Vol. 11, No. 2. P. 379-398. DOI: $10.15330 / \mathrm{cmp} \cdot 11.2 .379-398$

18. Nevanlinna R. Zur theorie der meromorphen funktionen. Acta Math., 1925. Vol. 46, No. 1-2. P. 1-99. (in German). DOI: 10.1007/BF02543858

19. Seneta E. Regularly Varying Functions. Lecture Notes in Math., Vol. 508. Berlin, Heidelberg: SpringerVerlag, 1976. 116 p. DOI: 10.1007/BFb0079658

20. Sheremeta M. N. Connection between the growth of the maximum of the modulus of an entire function and the moduli of the coefficients of its power series expansion. Izv. Vyssh. Uchebn. Zaved. Mat., 1967. Vol. 2, P. 100-108. (in Russian) 
21. Tu J., Chen Z.-X. Growth of solutions of complex differential equations with meromorphic coefficients of finite iterated order. Southeast Asian Bull. Math., 2009. Vol. 33, No. 1. P. 153-164.

22. Yang L., Value Distribution Theory. Berlin, Heidelberg: Springer-Verlag, 1993.269 p. DOI: $10.1007 / 978-3-662-02915-2$ 